\title{
Some Applications of the Katětov Order on Borel Ideals by
}

\author{
Nikodem MROŻEK
}

\author{
Presented by Tomasz EUCZAK
}

\begin{abstract}
Summary. We construct an embedding of the algebra $\mathcal{P}(\omega)$ /Fin into the family of summable ideals with the Katětov order. This construction will be used to solve two problems: about the relation between the Katětov order and the ideal Baire classes of functions, and about long chains of ideals alternately with and without the property of being a P-ideal.
\end{abstract}

1. Introduction. An ideal on the set $\omega$ of natural numbers is a family $\mathcal{I} \subset \mathcal{P}(\omega)$ (where $\mathcal{P}(\omega)$ denotes the power set of $\omega$ ) which is closed under taking subsets and finite unions. We denote by Fin the ideal of all finite subsets of $\omega$. We assume that all the ideals under consideration are proper $(\neq \mathcal{P}(\omega))$ and contain all finite sets.

Given two ideals $\mathcal{I}$ and $\mathcal{J}$ we write $\mathcal{I} \leq_{K} \mathcal{J}$ if there exists a function $f: \omega \rightarrow \omega$ such that $f^{-1}[A] \in \mathcal{J}$ whenever $A \in \mathcal{I}$. This preorder is called the Katětov order and was introduced by Katětov [4, 5].

Many topological and combinatorial properties could be described by finding a locally minimal (in the Katětov order) ideal among ideals having a given property (see [10], [12], [1] or [7]). In particular, Katětov investigated ideal convergence of sequences of continuous functions using this order. In [5] he proved that if $\mathcal{I} \leq_{K} \mathcal{J}$ then $\mathcal{B}_{1}^{I}(T) \subset \mathcal{B}_{1}^{J}(T)$ (where $\mathcal{B}_{1}^{I}(T)$ is the family of $\mathcal{I}$-Baire class one functions over a topological space $T$, see Section 2 for a formal definition). In the same article he asked about the converse implication:

Problem 1.1. If $\mathcal{B}_{1}^{I}(T) \subset \mathcal{B}_{1}^{J}(T)$ for any topological space $T$, does it follow that $\mathcal{I} \leq_{K} \mathcal{J}$ ?

2010 Mathematics Subject Classification: Primary 03E05; Secondary 03E15, 26A21.

Key words and phrases: Katětov order, P-ideal, Baire classes, summable ideal.

Received 20 January 2016; revised 17 June 2016.

Published online 11 July 2016. 
The answer can be deduced from [6] where the authors proved that $\mathcal{B}_{1}^{\mathcal{I}_{d}}(X)=\mathcal{B}_{1}^{\text {Fin }}(X)$ where $\mathcal{I}_{d}$ is the ideal of sets of asymptotic density zero and $X$ is a complete metric space. It is easy to prove that $\mathcal{I}_{d} \mathbb{Z}_{K}$ Fin, hence we have a negative answer to Katětov's problem. In Corollary 3.8 we give a stronger counterexample by showing that below any analytic P-ideal there is a family of size continuum of pairwise incomparable (in the Katětov order) ideals such that the Baire classes generated by them are equal.

In Section 4 we use the above construction to answer a question of Wilczyński. During the problem session at the 23th International Summer Conference on Real Functions Theory in Niedzica the following problem was formulated:

Problem 1.2. Does there exist, for any $n \in \omega$, a sequence of ideals

$$
\mathcal{I}_{0} \subset \mathcal{I}_{1} \subset \cdots \subset \mathcal{I}_{n}
$$

such that $\mathcal{I}_{i}$ is a P-ideal iff $i$ is even?

In Theorem 4.4 we give a positive answer to this question by producing even a transfinite sequence of ideals with this property.

2. Preliminaries. An ideal $\mathcal{I}$ is called dense if for any infinite set $A \subset \omega$ there exists an infinite set $B \subset A$ which belongs to $\mathcal{I}$.

An ideal $\mathcal{I}$ is a $P$-ideal if for every sequence $\left(A_{n}\right)_{n \in \omega}$ of sets from $\mathcal{I}$ there is $A \in \mathcal{I}$ such that $A_{n} \subset^{\star} A$, i.e. $A_{n} \backslash A \in$ Fin for all $n$.

By identifying sets of naturals with their characteristic functions, we can treat $\mathcal{P}(\omega)$ as the Cantor cube with the natural product topology and therefore we can assign the topological complexity to ideals of sets of integers. In particular, an ideal $\mathcal{I}$ is analytic if it is a continuous image of a $G_{\delta}$ subset of the Cantor space.

A map $\phi: \mathcal{P}(\omega) \rightarrow[0, \infty]$ is a submeasure on $\omega$ if

$$
\phi(\emptyset)=0, \quad \phi(A) \leq \phi(A \cup B) \leq \phi(A)+\phi(B),
$$

for all $A, B \subset \omega$. It is lower semicontinuous (lsc for short) if for all $A \subset \omega$ we have

$$
\phi(A)=\lim _{n \rightarrow \infty} \phi(A \cap\{0,1, \ldots, n-1\}) .
$$

For any lsc submeasure on $\omega$, let $\|\cdot\|_{\phi}: \mathcal{P}(\omega) \rightarrow[0, \infty]$ be the submeasure defined by

$$
\|A\|_{\phi}=\lim _{n \rightarrow \infty} \phi(A \backslash\{0,1, \ldots, n-1\}) .
$$

Let

$$
\operatorname{Exh}(\phi)=\left\{A \subset \omega:\|A\|_{\phi}=0\right\} .
$$

All analytic P-ideals were characterized by Solecki [11]. 
THEOREM 2.1. The following conditions are equivalent for an ideal $\mathcal{I}$ on $\omega$.

(1) $\mathcal{I}$ is an analytic P-ideal;

(2) $\mathcal{I}=\operatorname{Exh}(\phi)$ for some lsc submeasure $\phi$ on $\omega$.

It is easy to observe that:

FACT 2.2. For any lsc submeasure $\phi, \operatorname{Exh}(\phi)$ is dense iff $\lim _{n \rightarrow \infty} \phi(\{n\})$ $=0$.

For a function $g: \omega \rightarrow \mathbb{R}$ such that $\sum_{n \in \omega} g(n)=\infty$ the family

$$
\mathcal{I}_{g}=\left\{A \subset \omega: \sum_{n \in A} g(n)<\infty\right\}
$$

is an analytic P-ideal called a summable ideal generated by $g$.

Let $T$ be a topological Hausdorff space and $\mathcal{I}$ be an ideal on $\omega$. We say that a sequence $\left(x_{n}\right)_{n \in \omega}$ in $T$ is $\mathcal{I}$-convergent to $x \in T$ if

$$
\left\{n \in \omega: x_{n} \notin U\right\} \in \mathcal{I}
$$

for every open neighborhood $U$ of $x$.

We say that a sequence $\left(f_{n}: T \rightarrow \mathbb{R}\right)_{n \in \omega}$ of functions is pointwise $\mathcal{I}$ convergent if $\left(f_{n}(x)\right)_{n \in \omega}$ is $\mathcal{I}$-convergent for every $x \in T$.

Using this definition we can introduce ideal Baire classes of functions. We say that a function $f$ is of $\mathcal{I}$-Baire class one if it is an $\mathcal{I}$-pointwise limit of continuous functions. The family of all $\mathcal{I}$-Baire class one functions over a Hausdorff space $T$ is denoted by $\mathcal{B}_{1}^{\mathcal{I}}(T)$.

Laczkovich and Recław [8] proved the following theorem.

TheOREm 2.3. If $\mathcal{I}$ is a non-pathological analytic P-ideal and $T$ is a Hausdorff space, then $\mathcal{B}_{1}^{\mathcal{I}}(T)=\mathcal{B}_{1}(T)$.

The definition of a non-pathological analytic P-ideal is found in [8]; in particular, all summable ideals are non-pathological.

\section{Katětov's problem}

Theorem 3.1. Let $\mathcal{I}$ be a dense analytic P-ideal. There exists an embedding of the algebra $\mathcal{P}(\omega)$ /Fin into the family of summable ideals included in $\mathcal{I}$.

A weaker version of this theorem was proved independently by MezaAlcántara [10] and published in [2].

To prove this theorem we start with the construction of a family of ideals. Fix a dense analytic P-ideal $\mathcal{I}=\operatorname{Exh}(\phi)$ for some lsc submeasure $\phi$.

Let $\left(p_{n}\right)_{n \in \omega}$ be a sequence of natural numbers such that

(1) $p_{0}=0$,

(2) $\frac{p_{n}}{2\left(p_{0}+p_{1}+\cdots+p_{n-1}\right)+1}>2^{2^{n}}$, 
(3) $\phi(\{m\})<1 / 2^{2^{n+1}}$ for all $m>p_{0}+p_{1}+\cdots+p_{n-1}$.

The fulfillment of the third condition is possible by Fact 2.2 .

For all $n \geq 1$ let

$S_{n}=\left\{p_{0}+p_{1}+\cdots+p_{n-1}, p_{0}+p_{1}+\cdots+p_{n-1}+1, \ldots, p_{0}+p_{1}+\cdots+p_{n}\right\}$.

Obviously $\left\{S_{n}\right\}_{n \in \omega}$ is a partition of the naturals. For each $n$ define two measures $\phi_{n}^{0}$ and $\phi_{n}^{1}$ on $S_{n}$ by

$$
\phi_{n}^{0}(A)=\frac{|A|}{2^{2^{n+1}}}, \quad \phi_{n}^{1}(A)=\frac{|A|}{2^{2^{n}}} .
$$

For each infinite set $M \subset \omega$ define the ideal

$$
\mathcal{I}_{M}=\left\{A \subset \omega: \sum_{n \in M} \phi_{n}^{1}\left(A \cap S_{n}\right)<\infty, \sum_{n \in \omega \backslash M} \phi_{n}^{0}\left(A \cap S_{n}\right)<\infty\right\} .
$$

Lemma 3.2. For each infinite set $M$ the ideal $\mathcal{I}_{M}$ is a summable ideal contained in $\mathcal{I}$.

Proof. To prove the summability it is enough to observe that $\mathcal{I}_{M}$ is generated by the function

$$
f_{M}(i)= \begin{cases}1 / 2^{2^{n}} & \text { if } i \in S_{n} \text { and } n \in M \\ 1 / 2^{2^{n+1}} & \text { if } i \in S_{n} \text { and } n \notin M\end{cases}
$$

To justify the inclusion in $\mathcal{I}$ notice that $\phi \leq f_{M}$.

Lemma 3.3. Let $A, B$ be infinite subsets of $\omega$. If $B \subset^{\star} A$, then $\mathcal{I}_{A} \subset \mathcal{I}_{B}$.

Proof. This follows from the easy observation that $B \subset^{\star} A$ implies that $f_{B}(n) \leq f_{A}(n)$ for sufficiently large $n$.

Lemma 3.4. $\mathcal{I}_{A} \leq_{K} \mathcal{I}_{B}$ iff $B \subset^{\star} A$.

Proof. The implication $\Leftarrow$ follows from Lemma 3.3 and from the implication

$$
\mathcal{I} \subset \mathcal{J} \Rightarrow \mathcal{I} \leq_{K} \mathcal{J}
$$

To prove the converse, suppose that $B \backslash A$ is infinite and $\mathcal{I}_{A} \leq_{K} \mathcal{I}_{B}$. Hence there exists a function $f: \omega \rightarrow \omega$ such that $f^{-1}(I) \in \mathcal{I}_{B}$ for any $I \in \mathcal{I}_{A}$.

For each $n \in \omega$ we have two possibilities:

(a) $\left\{i \in S^{n}: f(i) \in \bigcup_{i>n} S_{i}\right\}$ has at least $2^{2^{n}}$ elements,

(b) $\left\{i \in S^{n}: f(i) \in \bigcup_{i<n} S_{i}\right\}$ has at least $p_{n} / 2$ elements.

One of them holds for infinitely many $n$ in $B \backslash A$. Let $N$ be the set of all such $n$. 
Suppose that $N$ consists of those $n$ for which (a) holds. For each $n \in N$ choose $D_{n} \subset S_{n}$ such that $\left|D_{n}\right|=2^{2^{n}}$ and $f\left(D_{n}\right) \subset \bigcup_{i \geq n} S_{i}$. Define

$$
E=\bigcup_{n \in \omega} f\left(D_{n}\right) \text {. }
$$

Notice that for each $n$,

$$
\sum_{m \in \omega} \phi_{m}^{0}\left(f\left(D_{n}\right) \cap S_{m}\right) \leq \frac{1}{2^{2^{n}}} .
$$

Hence $E \in \mathcal{I}_{A}$. On the other hand, for each $n \in N$ we have $\left|f^{-1}(E) \cap S_{n}\right|$ $\geq 2^{2^{n}}$, hence $\phi_{n}^{1}\left(f^{-1}(E) \cap S_{n}\right)=1$. Finally $f^{-1}(E) \notin \mathcal{I}_{B}$, a contradiction.

Suppose now that $N$ consists of those $n$ for which (b) holds. For each $n \in N$ choose $e_{n}$ such that $\left|f^{-1}\left(\left\{e_{n}\right\}\right) \cap S_{n}\right|>2^{2^{n}}$ (this is possible by the condition (1) on $\left(p_{n}\right)_{n}$ and the pigeonhole principle). Let $E \subset\left\{e_{n}\right\}_{n \in N}$ be such that

- $\left|E \cap S_{i}\right| \leq 1$ for all $i \in \omega$,

- $\left|f^{-1}(E) \cap S_{n}\right|>2^{2^{n}}$ for infinitely many $n \in N$.

The first condition guarantees that $E \in \mathcal{I}_{A}$. By the second condition $\phi_{n}^{1}\left(f^{-1}(E) \cap S_{n}\right) \geq 1$ for infinitely many $n \in N \subset B$. This implies that $f^{-1}(E) \notin \mathcal{I}_{B}$, a contradiction.

Proof of Theorem 3.1. Consider the mapping

$$
\mathcal{P}(\omega) / \text { Fin } \ni M \mapsto \mathcal{I}_{M} .
$$

It is well defined since if $A \triangle B \in$ Fin, then $f_{A}=f_{B}$ almost everywhere, hence $\mathcal{I}_{A}=\mathcal{I}_{B}$. The fact that it is an embedding follows from Lemma 3.4 .

DEFinition 3.5. We call a family $\left\{\mathcal{I}_{\alpha}\right\}_{\alpha}$ of ideals an $\leq_{K^{-}}$antichain if

$$
\mathcal{I}_{\alpha} \leq_{K} \mathcal{I}_{\alpha^{\prime}} \Leftrightarrow \alpha=\alpha^{\prime}
$$

Notice that this definition is different from the classical definition of the antichain in a Boolean algebra, where there is no element of the algebra smaller than two distinct elements of the antichain. Such a definition would be too strong since for any two ideals $\mathcal{I}, \mathcal{J}$ we have $\mathcal{I} \oplus \mathcal{J} \leq_{K} \mathcal{I}, \mathcal{J}$ where $\mathcal{I} \oplus \mathcal{J}$ is the disjoint union of $\mathcal{I}$ and $\mathcal{J}$.

Hrušák and García Ferreira [3] showed that below any dense ideal there

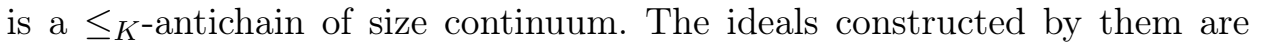
generated by maximal almost disjoint families, so by the result of Mathias [9] they are not analytic. Since there exists an almost disjoint family in $\mathcal{P}(\omega)$ of cardinality continuum, we get the following corollary:

Corollary 3.6. Let $\mathcal{I}$ be a dense analytic P-ideal. Below $\mathcal{I}$, there exists $a \leq_{K}$-antichain of cardinality continuum consisting of summable ideals. 
Here we cannot replace a dense analytic P-ideal by any dense ideal. Recall that if $\mathcal{A}$ is an almost disjoint family in $\mathcal{P}(\omega)$ then

$$
\{B \subset \omega: A \cap B \text { is infinite only for finitely many } A \in \mathcal{A}\}
$$

is an ideal.

THEOREM 3.7. If $\mathcal{I}$ is an ideal generated by an almost disjoint family $\mathcal{A}$, and $\mathcal{J}$ is any dense $P$-ideal, then $\mathcal{J} \mathbb{L}_{K} \mathcal{I}$.

Proof. Suppose that $\mathcal{J} \leq_{K} \mathcal{I}$. Let $f: \omega \rightarrow \omega$ be a function from the definition of the Katětov order.

Choose a countable family $\left\{A_{n}\right\}_{n \in \omega} \subset \mathcal{A}$. Let $N$ be the set of all $n$ such that $f\left(A_{n}\right)$ is infinite.

Suppose that $N$ is infinite. Since $\mathcal{J}$ is a dense ideal, for each $n$ we can choose an infinite set $E_{n} \subset f\left(A_{n}\right)$ and $E_{n} \in \mathcal{J}$. Let $E \in \mathcal{J}$ be such that $E_{n} \subset^{\star} E$ for each $n \in N$. Notice that $f^{-1}(E) \cap A_{n}$ is infinite for each $n \in N$, hence $f^{-1}(E) \notin \mathcal{J}$ - a contradiction.

Suppose that $N$ is finite. For each $n \in \omega \backslash N$ choose $e_{n} \in f\left(A_{n}\right)$ such that $f^{-1}\left(e_{n}\right)$ is infinite. Let $E \subset\left\{e_{n}\right\}_{n \in \omega}$ be an infinite set such that $E \in \mathcal{J}$. Observe that $f^{-1}(E) \cap A_{n}$ is infinite for each $n \in N$. Hence $f^{-1}(E) \notin \mathcal{I}$ a contradiction.

Finally, as a corollary from Theorem 2.3 and Corollary 3.6 we get the following answer to Katětov's problem:

Corollary 3.8. Let $\mathcal{I}$ be a dense analytic P-ideal. There exists a family $\left\{\mathcal{I}_{\alpha}\right\}_{\alpha<\mathfrak{c}}$ of ideals pairwise incomparable in the Katètov order such that $\mathcal{I}_{\alpha} \leq_{K} \mathcal{I}$ and $\mathcal{B}_{1}^{\mathcal{I}_{\alpha}}(T)=\mathcal{B}_{1}(T)$ for each $\alpha<\mathfrak{c}$ and every Hausdorff space $T$.

4. Wilczyński's problem. Recall the definition of the bounded number $\mathfrak{b}$ :

$$
\mathfrak{b}=\min \left\{|F|: F \subset \omega^{\omega} \text { and } \forall_{g \in \omega^{\omega}} \exists_{f \in F} \forall_{n \in \omega} \exists_{m>n} g(m)<f(m)\right\} .
$$

Recall that $\aleph_{0}<\mathfrak{b} \leq \mathfrak{c}$. Obviously CH implies $\mathfrak{b}=\mathfrak{c}$, but in the Cohen model we have $\aleph_{1}=\mathfrak{b}<\mathfrak{c}$.

Definition 4.1. Let $\gamma$ be an ordinal number. We call a family $\left\{\mathcal{I}_{\alpha}\right\}_{\alpha<\gamma}$ of ideals an increasing $\leq_{K}$-chain if for any $\alpha, \alpha^{\prime}<\gamma$,

$$
\alpha \leq \alpha^{\prime} \Leftrightarrow \mathcal{I}_{\alpha} \leq_{K} \mathcal{I}_{\alpha^{\prime}} .
$$

Since there are increasing chains of length $\mathfrak{b}$ in $\mathcal{P}(\omega) /$ Fin, we get the following corollary from Theorem 3.1 .

Corollary 4.2. Let $\mathcal{I}$ be an analytic P-ideal. Below $\mathcal{I}$, there exists an increasing $\leq_{K}$-chain of length $\mathfrak{b}$ of summable ideals.

In Theorem 4.4 we will use this $\leq_{K}$-chain to answer Wilczyński's problem, but first we need the following proposition: 
Proposition 4.3. If $\mathcal{I}_{0} \subset \mathcal{I}_{1} \subset \cdots \subset \mathcal{I}_{n} \subset \cdots$ is a strictly increasing sequence of ideals, then $\bigcup_{n \in \omega} \mathcal{I}_{n}$ is an ideal which is not a P-ideal.

Proof. It is easy to observe that $\mathcal{I}=\bigcup_{n \in \omega} \mathcal{I}_{n}$ is an ideal.

We now show that $\mathcal{I}$ is not a P-ideal. Since $\left(\mathcal{I}_{n}\right)_{n \in \omega}$ is a strictly increasing sequence of ideals, for each $n \in \omega$ we can choose $A_{n} \in \mathcal{I}_{n+1} \backslash \mathcal{I}_{n}$. Suppose that $A$ is such that $A_{n} \backslash A$ is finite for each $n \in \omega$. Fix $n \in \omega$. Since $A_{n} \notin \mathcal{I}_{n}$, also $A \notin \mathcal{I}_{n}$. Hence $A \notin \mathcal{I}$.

Let $\alpha=\lambda+n$ be an ordinal, where $n \in \omega$ and $\lambda$ is a limit number or zero. We call $\alpha$ an even [odd] ordinal if $n$ is even [odd].

Theorem 4.4. Let $\mathcal{I}$ be a dense analytic P-ideal. There exists a sequence $\left(\mathcal{K}_{\alpha}\right)_{\alpha<\mathfrak{b}}$ of dense analytic ideals such that

(1) $\mathcal{K}_{\alpha} \subset \mathcal{K}_{\beta} \subset \mathcal{I}$ for $\alpha<\beta<\mathfrak{b}$,

(2) $\mathcal{K}_{\alpha}$ is a P-ideal iff $\alpha$ is even.

Proof. Let $\left\{\mathcal{I}_{\alpha}\right\}_{\alpha<\mathfrak{b}}$ be an increasing $\leq_{K}$-chain of ideals from Corollary 4.2 (constructed as in the proof of Theorem 3.1). By Lemma 3.3, if $\alpha<\beta<\mathfrak{b}$, then $\mathcal{I}_{\alpha} \subset \mathcal{I}_{\beta}$.

For $\alpha<\mathfrak{b}$ define ideals

$$
\mathcal{J}_{\alpha}= \begin{cases}\mathcal{I}_{\omega \cdot \alpha} & \text { if } \alpha \text { is an even ordinal, } \\ \bigcup_{n \in \omega} \mathcal{I}_{\omega \cdot \alpha+n} & \text { if } \alpha \text { is an odd ordinal. }\end{cases}
$$

Since $\left(\mathcal{I}_{\alpha}\right)_{\alpha<\mathfrak{b}}$ is an increasing sequence of ideals, the sequence $\left(\mathcal{J}_{\alpha}\right)_{\alpha<\mathfrak{b}}$ is also increasing. Conclusion (2) holds by Proposition 4.3 .

\section{References}

[1] P. Barbarski, R. Filipów, N. Mrożek, and P. Szuca, When does the Katětov order imply that one ideal extends the other?, Colloq. Math. 130 (2013), 91-102.

[2] O. Guzmán-González and D. Meza-Alcántara, Some structural aspects of the Katětov order on Borel ideals, Order, to appear.

[3] M. Hrušák and S. García Ferreira, Ordering MAD families à la Katětov, J. Symbolic Logic 68 (2003), 1337-1353.

[4] M. Katětov, Products of filters, Comment. Math. Univ. Carolin. 9 (1968), 173-189.

[5] M. Katětov, On descriptive classes of functions, in: Theory of Sets and Topology (in honour of Felix Hausdorff, 1868-1942), Deutsch. Verlag Wiss., Berlin, 1972, 265-278.

[6] P. Kostyrko, T. Šalát, and W. Wilczyński, $\mathcal{I}$-convergence, Real Anal. Exchange 26 $(2000 / 01), 669-685$.

[7] A. Kwela, A note on a new ideal, J. Math. Anal. Appl. 430 (2015), 932-949.

[8] M. Laczkovich and I. Recław, Ideal limits of sequences of continuous functions, Fund. Math. 203 (2009), 39-46.

[9] A. R. D. Mathias, Happy families, Ann. Math. Logic 12 (1977), 59-111.

[10] D. Meza-Alcántara, Ideals and filters on a countable set, Ph.D. thesis, Univ. Nacional Autónoma de México, 2009. 
[11] S. Solecki, Analytic ideals and their applications, Ann. Pure Appl. Logic 99 (1999), $51-72$.

[12] S. Solecki, Filters and sequences, Fund. Math. 163 (2000), 215-228.

Nikodem Mrożek

Institute of Mathematics

Faculty of Mathematics, Physics, and Informatics

University of Gdańsk

Wita Stwosza 57

80-952 Gdańsk, Poland

E-mail: nmrozek@mat.ug.edu.pl 